\title{
A Preliminary Survey of the Distribution of Segmented Filamentous Bacteria in the Porcine Gastrointestinal Tract
}

\author{
Łukasz Grześkowiak ${ }^{1}$ · Beatriz Martínez-Vallespín ${ }^{1} \cdot$ Jürgen Zentek $^{1} \cdot$ Wilfried Vahjen ${ }^{1}$
}

Received: 25 January 2021 / Accepted: 18 August 2021 / Published online: 3 September 2021

(c) The Author(s) 2021

\begin{abstract}
Segmented filamentous bacteria (SFB) are present in various animal species including pigs. The aim of this work was to analyze the occurrence of SFB in different parts of the gastrointestinal tract of piglets of different ages. A total of 377 DNA extracts from stomach, jejunum, ileum, cecum and colon digesta, and from feces collected on different time points, originating from 155 animals, were screened by qPCR method with primers specific for the SFB. SFB sequences were detected in 74 of 377 samples (19.6\%) from 155 animals in total. SFB were most abundant in ileum (50.0\%), cecum (45.0\%), and colon (37.0\%), followed by feces (14.6\%). SFB prevalence in sows was $12.9 \%(13 / 101)$ and $75.9 \%(41 / 54)$ in individual piglets. Of the 41 SFB-positive piglets, only two samples were from pre-weaning animals, while the rest of samples originated from post-weaning piglets. SFB sequences are abundant in post-weaning piglets, but not in suckling or adult animals. They are most abundant in the ileum and cecum of pigs. Further studies are warranted to reveal the role of SFB in pigs.
\end{abstract}

\section{Introduction}

The gut microbiota of pigs is characterized by rapid development after birth with certain bacteria temporarily dominating the intestinal tract. Segmented filamentous bacteria (SFB) are Gram-positive, spore-forming bacteria which have been detected in a wide range of mammals, birds, humans, and also in insects [1,2]. In rodents such as mice and rats, SFB are found predominantly around weaning and persist only shortly afterward $[3,4]$. The reason for the age-dependence in SFB colonization is not well understood yet. Although the degree of SFB colonization differs within species, they must be considered as indigenous bacteria of the intestine [5].

Data from light and scanning electron microscopy demonstrate that the main site of SFB colonization in mice, rats, chickens, dogs, and pigs is the ileum, where SFB attach to the intestinal epithelium without provoking an immune response $[1,6,7]$. The unique symbiotic lifestyle of SFB is related to their limited metabolism capacity. For instance, SFB lack the ability to synthesize most amino acids, vitamins, or nucleotides [8] and therefore, they seem to be highly dependent on nutrients and co-factors derived from

Łukasz Grześkowiak

lukasz.grzeskowiak@fu-berlin.de

1 Institute of Animal Nutrition, Freie Universität Berlin, Berlin, Germany their host [9]. Since SFB depend on critical growth factors, an in vitro cultivation attempts of SFB remain a challenge [10].

Segmented filamentous bacteria (SFB) are gaining attention due to their role in induction of adaptive and innate immunity in mice and rats [11]. However, recent reports demonstrate that SFB could also be associated with certain autoimmune, chronic inflammatory diseases in mouse models [12]. Thus, their exact role on host health needs still to be clarified.

Although SFB have been widely characterized in the laboratory rodents, still little is known about SFB in pigs. Therefore, the aim of this work was to perform a preliminary survey to detect and analyze the occurrence of SFB in different parts of the gastrointestinal tract of piglets of different age and in the sows' feces.

\section{Materials and Methods}

\section{DNA Extracts and qPCR}

A total of 377 existing DNA extracts from stomach, jejunum, ileum, cecum and colon digesta, and from feces, originating from 155 animals and 11 different feeding trials, all performed at our animal facility station were collected from the own DNA storage. The samples used in the current study 
are from the DNA storage and no live animals were used in the current study. All the samples originated from standardized feeding animal trials with identical standard animal husbandry conditions (sows kept in barns; suckling piglets with their mothers until weaning; post-weaning pigs kept in pairs in pens). Animals were fed standard wheat-based diets, appropriate for their age. Since it was a screening study, to avoid the impact of specific dietary treatments on the SFB prevalence, only the DNA from animals assigned to control groups was used (Table 1). The DNA has been obtained using extraction methods with different commercial kits, specifically the QIAamp DNA stool Mini kit (Qiagen, Hilden, Germany) and the NucleoSpin DNA Stool (Macherey-Nagel GmbH and Company KG, Düren, Germany); therefore, the further obtained data were considered as semiquantitative results. The DNA extracts were subjected to SFB screening by qPCR method with the following primer set: 779F (5'-TGTGGGTTGTGAATAACAAT-3'), 1008R (5'-GCGAGCTTCCCTCATTACAAGG-3') [13, 14]. The PCR conditions were as follows: $1 \times 15 \mathrm{~min} ; 40 \times 15 \mathrm{~s} 95^{\circ} \mathrm{C}$, 60 s $56{ }^{\circ} \mathrm{C}, 60$ s $72{ }^{\circ} \mathrm{C} ; 1 \times$ ramp from 56 to $95^{\circ} \mathrm{C}$. The master mix consisted of Brilliant II SYBR Green QPCR Master Mix with Low ROX (Stratagene, San Diego, CA, USA). Real-time cycler MX3005 and MX3000 (Stratagene) were used for PCR amplification and fluorescent data collection. After confirmation of correct dissociation temperature, samples were identified as positive, if the respective cT-value lay in range of the calibration curve samples (cut-off at 35 cycles).

\section{Statistical Analysis}

The DNA extracts were not normalized for identical DNA concentration. Instead, inverse cT values from amplification data were used to approximate semi-quantitative distributions of bacteria in sample extracts. As unequal sample sizes were present in the data sets; the non-parametric Kruskal-Wallis test and subsequent Mann-Whitney $U$ test were used to denote significant differences. Significance was considered at $P \leq 0.05$. Data were analyzed in SPSS 24.0.0.0 (IBM, SPSS Statistics, Chicago, IL, USA).

\section{Results and Discussion}

The prevalence of SFB among different animal species seems to be ubiquitous. SFB have been detected in a broad range of mammals like monkeys, horses, cattle, pigs, dogs, mice, rats, and in birds [1-5]. Interestingly, previous attempts to colonize germ-free rats or germ-free mice with SFB obtained from unrelated hosts have failed, indicating that SFB are host specific [15]. Such unique host specificity down to species level may be due to the intimate association of SFB with host epithelial surfaces in the gut. Moreover, SFB have been found to colonize certain species in an agedependent manner [5, 6]. Here, we show that the prevalence of SFB in pigs is also age dependent. SFB prevalence in the individual piglets was $75.9 \%$ (41/54). Specifically, SFB were almost undetectable in suckling piglets $(0-5.3 \%)$, while their abundance increased after weaning (28.6-63.2\%) and diminished drastically in adult pigs (12.9\%) (Fig. 1). Further, division of all the analyzed samples (intestinal digesta and feces, different sampling time points) according to piglets or sows yielded a percentage of 22.1\% (61/276) SFB-positive samples in piglets and $12.9 \%$ (13/101) in sows (Fig. 2). Of the 61 all SFB-positive samples in piglets, only two samples were from a single pre-weaning animal, while the rest came from post-weaning piglets (Fig. 2). Weaning in piglets, which is characterized by transition from sow milk to exclusive solid feeding and separation from mother sows, usually occurs between third and fourth week of life [16]. Our findings are consistent with a previous report on the SFB prevalence in pigs [6]. Also in mice and rats, SFB are almost absent in neonates, while they arise after weaning and diminish again in adulthood $[3,4,17,18]$. Therefore, it is becoming clear that SFB are more commonly detected in younger versus older animals. However, the rationale for that observation is not understood. In the offspring, the colonization with SFB may depend on numerous factors such as the health status of a mother, and thus timing and persistence of SFB colonization may also be related to the transfer of maternal SFB and certain key features like immune agents. In mammalian neonates including suckling piglets, the specific composition of colostrum and milk, enriched in IgA
Table 1 Information on the origin of DNA extracts for the study

\begin{tabular}{lrl}
\hline Item & $N$ & Note \\
\hline Total sample number & 377 & From a total of 155 animals \\
Number of trials & 11 & Only control groups were used \\
Intestinal location & 6 & $\begin{array}{c}\text { Stomach }(n=22), \text { jejunum }(n=33), \text { ileum }(n=22), \\
\text { cecum }(n=20), \text { colon }(n=54), \text { feces }(n=226)\end{array}$ \\
Piglet samples & 276 & Suckling piglets $(n=107)$, post-weaned piglets $(n=169)$ \\
Sow samples & 101 & All feces \\
Age groups & 9 & From 10- to 56-day-old piglets; sows of varying age \\
\hline
\end{tabular}




\begin{tabular}{lccc}
\hline Age (week) & SFB-positive samples & Total animals & \% SFB-positive \\
\hline 1 & 1 & 24 & 4.2 \\
2 & 0 & 24 & 0 \\
3 & 1 & 19 & 5.3 \\
Subtotal (1-3 week) & 2 & 67 & 3.0 \\
\hline 4 & 6 & 21 & 28.6 \\
5 & 3 & 9 & 33.3 \\
6 & 4 & 10 & 40.0 \\
7 & 12 & 19 & 63.2 \\
8 & 24 & 34 & 70.6 \\
Subtotal (4-8 week) & 49 & 93 & 52.7 \\
Adult & 13 & 101 & 12.9 \\
\hline
\end{tabular}

Fig. 1 Distribution of animals in which at least one sample (intestinal digesta or feces) was positive for SFB, as analyzed by qPCR. Horizontal line between Subtotal (1-3 week) and 4th week of age indicates weaning and thus separates suckling from weaning piglets

\begin{tabular}{|c|c|c|c|}
\hline Age (week) & SFB-positive samples & Total sample number & $\%$ SFB-positive \\
\hline $1^{\mathrm{a}, \mathrm{b}}$ & 1 & 24 & 4.2 \\
\hline $2^{\mathrm{a}}$ & 0 & 64 & 0 \\
\hline $3^{\mathrm{a}, \mathrm{b}}$ & 1 & 19 & 5.3 \\
\hline Subtotal (1-3 week) & 2 & 107 & 1.9 \\
\hline $4^{\mathrm{a}, \mathrm{b}}$ & 6 & 41 & 14.6 \\
\hline $5^{\mathrm{a}, \mathrm{b}}$ & 3 & 14 & 21.4 \\
\hline $6^{\mathrm{a}, \mathrm{b}, \mathrm{c}}$ & 4 & 15 & 26.7 \\
\hline $7^{b, c}$ & 12 & 37 & 32.4 \\
\hline $8^{c}$ & 34 & 62 & 54.8 \\
\hline Subtotal (4-8 week) & 59 & 169 & 34.9 \\
\hline Adult $^{\mathrm{a}, \mathrm{b}}$ & 13 & 101 & 12.9 \\
\hline \multicolumn{4}{|l|}{ Gut segment } \\
\hline Stomach $^{\mathrm{a}}$ & 1 & 22 & 4.6 \\
\hline Jejunum $^{\mathrm{a}}$ & 0 & 33 & 0 \\
\hline Ileum $^{c}$ & 11 & 22 & 50.0 \\
\hline Cecum $^{c}$ & 9 & 20 & 45.0 \\
\hline Colon ${ }^{b, c}$ & 20 & 54 & 37.0 \\
\hline Feces $^{\mathrm{a}, \mathrm{b}}$ & 33 & 226 & 14.6 \\
\hline
\end{tabular}

Fig. 2 Distribution of SFB-positive samples in the intestinal digesta and feces of pigs according to age and different intestinal segments of pigs, as analyzed by qPCR. Horizontal line between Subtotal
(1-3 week) and 4th week of age indicates weaning and thus separates suckling from weaning piglets. ${ }^{\mathrm{a}, \mathrm{b}, \mathrm{c}}$ Different superscripts denote significant differences (\% SFB-positive) within a column $(P \leq 0.05)$ 
and other antimicrobial agents, may prevent the SFB from establishing a successful colonization in the gut. Following the weaning period where the cessation of milk ingestion occurs, may open an opportunity for SFB to colonize certain gut niches, as previously investigated in mice and their pups [17]. Even though the SFB abundance increases after weaning, not all weaned piglets are colonized with SFB (Figs. 1, 2). The rationale for differences in colonization between the individual animals is not known yet. Another phenomenon is related to a low abundance of SFB among adult animals including pigs (Fig. 1). When and why the SFB almost vanish when the animal gets older needs further research.

It is known that certain bacteria are able to only temporarily colonize the gut of pigs. For instance, a very short time window of about one week after birth for colonization by Clostridioides difficile, including toxin-producing ribotypes, can be observed in healthy neonatal piglets $[19$, 20]. Most probably, $C$. difficile benefits from a yet immature developing gut microbiota. In healthy suckling piglets, the concentration of $C$. difficile diminishes from the second week onward and the spores or its toxins are rarely detected in weaned or adult pigs $[19,20]$. Similarly, various porcine pathogenic Escherichia coli strains show different windows of opportunity for colonization and infection in suckling or post-weaning pigs $[21,22]$. Whether the phenomenon of colonization resistance is true for SFB is not clear yet, however studies on $C$. difficile and $E$. coli could provide some useful considerations.

Moreover, SFB have been found to induce local immune response in murine gut, specifically activating intestinal $\operatorname{IgA}$ or $\mathrm{CD} 4+\mathrm{Th}_{17}$ cells $[23,24]$. The specific strengthening of the host immune system against other commensal bacteria by SFB may help them to compete for niche with other microbes and establish temporal colonization. It seems that the local response of the host does not eliminate the SFB from the gut but only prevents its overgrowth, keeping the SFB immune potential to a minimum necessary need to prevent gut epithelial invasion by pathogens.

Our study provides insight into the topographical distribution of SFB in the intestinal digesta of piglets (Fig. 2). Since we did not perform any dissections of the sows, the distribution of SFB in the digesta of intestinal segments of adult pigs still needs to be characterized. We found that the SFB were most abundant in ileum (50.0\%), followed by cecum $(45.0 \%)$ and colon (37.0\%) digesta and feces (14.6\%), while they were not detected in the jejunum digesta of piglets (Fig. 2). Previous publications also report the highest abundance of SFB in the ileum of piglets, mice and rats, and in the ileum and cecum of poultry $[5,6,25]$. It seems that the prevalence of SFB in gut segments proximal to the ileum is very low in piglets [6]. Specific physiological conditions in the ileum, such as lower oxygen level, slower transit time, and lower mucus secretion compared to jejunum, may help the SFB attach to the epithelium and establish a temporary population.

Segmented filamentous bacteria (SFB) are spore-forming bacteria that release spores from vegetative cells anchored in the gut epithelium, as previously suggested [1]. The transmission of SFB spores to the offspring is probably via the fecal-oral route. The ingested spores must persist in the gut until conditions are favorable for germination. The localization of SFB spores in the porcine gut before germination has not been confirmed. One could speculate that the SFB spores persist attached in the ileum in which they sense specific factors coming from the jejunum to germinate and release vegetative cells. Based on the total bacterial DNA extracted and primers used, it is not possible to distinguish between vegetative cells and spores of SFB. Finding the proper growth factors would allow to develop suitable media and conditions for in vitro SFB growth.

Segmented filamentous bacteria (SFB) are abundant in post-weaning piglets, but not in suckling or adult animals. SFB are most prominently detected in the ileum and cecum. The presence of SFB depends on unknown parameters during the development of a piglet, e.g., sow milk, solid feeding, immune-related factors, and/or indigenous microbiota. Further studies are warranted to reveal the role of SFB in pigs.

Funding Open Access funding enabled and organized by Projekt DEAL. This study was financially supported by the DSM Nutritional Products, Basel, Switzerland.

\section{Declarations}

Conflict of interest Authors declare no conflict of interest to the present study.

Ethical Approval The studies from which the DNA extracts originated were previously approved by the animal welfare authority the Landesamt für Gesundheit und Soziales, Berlin (Registration numbers: G0293/09, G0284/11, G281/13, G0389/12, G0347/09). Collection of the fecal samples did not require ethical committee approval.

Open Access This article is licensed under a Creative Commons Attribution 4.0 International License, which permits use, sharing, adaptation, distribution and reproduction in any medium or format, as long as you give appropriate credit to the original author(s) and the source, provide a link to the Creative Commons licence, and indicate if changes were made. The images or other third party material in this article are included in the article's Creative Commons licence, unless indicated otherwise in a credit line to the material. If material is not included in the article's Creative Commons licence and your intended use is not permitted by statutory regulation or exceeds the permitted use, you will need to obtain permission directly from the copyright holder. To view a copy of this licence, visit http://creativecommons.org/licenses/by/4.0/. 


\section{References}

1. Klaasen HLBM, Koopman JP, Poelma FGJ, Beynen AC (1992) Intestinal, segmented, filamentous bacteria. FEMS Microbiol Lett 88:165-179. https://doi.org/10.1016/0378-1097(92)90801-T

2. Chen H, Wang L, Wang X et al (2019) Distribution and strain diversity of immunoregulating segmented filamentous bacteria in human intestinal lavage samples. Microb Ecol. https://doi.org/ 10.1007/s00248-019-01441-4

3. Davis CP, Savage DC (1974) Habitat, succession, attachment, and morphology of segmented, filamentous microbes indigenous to the murine gastrointestinal tract. Infect Immun 10:948-956. https://doi.org/10.1128/iai.10.4.948-956.1974

4. Koopman JP, Stadhouders AM, de Boer H (1987) The attachment of filamentous segmented micro-organisms to the distal ileum wall of the mouse: a scanning and transmission electron microscopy study. Lab Anim 21:48-52. https://doi.org/10.1258/0023677877 80740743

5. Klaasen HLBM, Koopman JP, Van Den Brink ME et al (1993) Intestinal, segmented, filamentous bacteria in a wide range of vertebrate species. Lab Anim 27:141-150. https://doi.org/10.1258/ 002367793780810441

6. Sanford SE (1991) Light and electron microscopic observations of a segmented filamentous bacterium attached to the mucosa of the terminal ileum of pigs. J Vet Diagnostic Investig 3:328-333. https://doi.org/10.1177/104063879100300410

7. Blumershine RV, Savage DC (1977) Filamentous microbes indigenous to the murine small bowel: a scanning electron microscopic study of their morphology and attachment to the epithelium. Microb Ecol 4:95-103. https://doi.org/10.1007/BF02014280

8. Kuwahara T, Ogura Y, Oshima K et al (2011) The lifestyle of the segmented filamentous bacterium: a non-culturable gut-associated immunostimulating microbe inferred by whole-genome sequencing. DNA Res 18:291-303. https://doi.org/10.1093/dnares/dsr022

9. Sczesnak A, Segata N, Qin X et al (2011) The genome of Th17 cell-inducing segmented filamentous bacteria reveals extensive auxotrophy and adaptations to the intestinal environment. Cell Host Microbe 10:260-272. https://doi.org/10.1016/j.chom.2011. 08.005

10. Schnupf P, Gaboriau-Routhiau V, Gros M et al (2015) Growth and host interaction of mouse segmented filamentous bacteria in vitro. Nature 520:99-103. https://doi.org/10.1038/nature14027

11. Chung H, Pamp SJ, Hill JA et al (2012) Gut immune maturation depends on colonization with a host-specific microbiota. Cell 149:1578-1593. https://doi.org/10.1016/j.cell.2012.04.037

12. Wu H-J, Ivanov II, Darce J et al (2010) Gut-residing segmented filamentous bacteria drive autoimmune arthritis via T helper 17 cells. Immunity 32:815-827. https://doi.org/10.1016/j.immuni. 2010.06.001

13. Snel J, Heinen PP, Blok HJ et al (1995) Comparison of 16S rRNA sequences of segmented filamentous bacteria isolated from mice, rats, and chickens and proposal of "Candidatus Arthromitus."
Int J Syst Bacteriol 45:780-782. https://doi.org/10.1099/00207 713-45-4-780

14. Urdaci MC, Regnault B, Grimont PAD (2001) Identification by in situ hybridization of segmented filamentous bacteria in the intestine of diarrheic rainbow trout (Oncorhynchus mykiss). Res Microbiol 152:67-73. https://doi.org/10.1016/S0923-2508(00) 01169-4

15. Tannock GW, Miller JR, Savage DC (1984) Host specificity of filamentous, segmental microorganisms adherent to the small bowel epithelium in mice and rats. Appl Environ Microbiol 47:441-442. https://doi.org/10.1128/aem.47.2.441-442.1984

16. Humphrey B, Zhao J, Faris R (2019) Review: link between intestinal immunity and practical approaches to swine nutrition. Animal 13:2736-2744. https://doi.org/10.1017/S1751731119001861

17. Jiang HQ, Bos NA, Cebra JJ (2001) Timing, localization, and persistence of colonization by segmented filamentous bacteria in the neonatal mouse gut depend on immune status of mothers and pups. Infect Immun 69:3611-3617. https://doi.org/10.1128/IAI. 69.6.3611-3617.2001

18. Savage DC (1969) Localization of certain indigenous microorganisms on the ileal villi of rats. J Bacteriol 97:1505-1506. https:// doi.org/10.1128/jb.97.3.1505-1506.1969

19. Grześkowiak $Ł$, Zentek J, Vahjen W (2016) Determination of the extent of clostridium difficile colonisation and toxin accumulation in sows and neonatal piglets. Anaerobe 40:5-9. https://doi.org/10. 1016/j.anaerobe.2016.04.012

20. Grześkowiak Dadi, Zentek Vahjen (2019) Developing gut microbiota exerts colonisation resistance to clostridium (syn. clostridioides) difficile in piglets. Microorganisms 7:218. https://doi.org/ 10.3390/microorganisms7080218

21. Goswami PS, Friendship RM, Gyles CL et al (2011) Preliminary investigations of the distribution of Escherichia coli 0149 in sows, piglets, and their environment. Can J Vet Res 75:57-60

22. Luppi A (2017) Swine enteric colibacillosis: diagnosis, therapy and antimicrobial resistance. Porc Heal Manag 3:1-18. https://doi. org/10.1186/s40813-017-0063-4

23. Ivanov II, Atarashi K, Manel $\mathrm{N}$ et al (2009) Induction of intestinal Th17 cells by segmented filamentous bacteria. Cell 139:485-498. https://doi.org/10.1016/j.cell.2009.09.033

24. Klaasen HLBM, Van der Heijden PJ, Stok W et al (1993) Apathogenic, intestinal, segmented, filamentous bacteria stimulate the mucosal immune system of mice. Infect Immun 61:303-306. https://doi.org/10.1128/iai.61.1.303-306.1993

25. Yin Y, Wang Y, Zhu L et al (2013) Comparative analysis of the distribution of segmented filamentous bacteria in humans, mice and chickens. ISME J 7:615-621. https://doi.org/10.1038/ismej. 2012.128

Publisher's Note Springer Nature remains neutral with regard to jurisdictional claims in published maps and institutional affiliations. 